\title{
Delignification of Empty Fruit Bunch (EFB) using Low Transition Temperature Mixtures (LTTMs): A Review
}

\author{
Yiin Chung Loong ${ }^{1, a}$, Suzana Yusup ${ }^{2, b^{*}}$, Yoshimitsu Uemura ${ }^{3, c}$ \\ 1,2,3 Biomass Processing Lab, Center of Biomass and Biofuels Research, MOR Green Technology, \\ Chemical Engineering Department, Universiti Teknologi PETRONAS, Bandar Seri Iskandar, 31750 \\ Tronoh, Perak, Malaysia. \\ aychungloong2009@gmail.com, bdrsuzana_yusuf@petronas.com.my, \\ cyoshimitsu_uemura@petronas.com.my
}

Keywords: LTTMs, EFB, microwave irradiation, solvation behaviour, delignification

\begin{abstract}
Biomass processing using low transition temperature mixtures (LTTMs) has the potential to become a sustainable alternative resource for production of raw materials and fuels with a neutral carbon dioxide balance. The state of art for customizing the physicochemical behaviour of these new green solvents by a prudent selection of the constituents' nature and ratio through microwave irradiation is presented in this work. The impact of the following parameters, namely type of malic acid, molar ratio of malic acid to natural salt, water content and temperature of treatment are related to the solvation behaviour of LTTMs. An overview of the conditions for the highest efficiency in the delignification of empty fruit bunch (EFB) are described.
\end{abstract}

\section{Introduction}

Biomass utilization stands out to be having the most potential and feasible option in harnessing its energy. Nilsen et al. (2007) highlighted that unlike other renewable sources of energy (i.e. solar, wind) which only generates heat and power, biomass can be converted to liquid, solid and gaseous fuels [1]. The components in lignocellulosic biomass such as lignin, cellulose and hemiscellulose are assembled in a complex three-dimensional structure which lead to resistant against chemicals and microbial attack that makes it very difficult to hydrolyze. An effective dissociation of these components and their subsequent separation is needed for the production of high value products from lignocellulosic biomass [2].

In future bio-economy, finding a suitable solvent for lignocellulosic biomass is becoming crucial for biofuels processing. However current processes to convert lignocellulosic into useful raw materials are highly inefficient. Even the most efficient process such as the production of paper from wood, show a biomass utilization of only $35 \%$ [3]. The implementation of the biorefinery concept is challenging due to technological limitations. Therefore, an economic feasibility of such industries is still questionable. Conventional methods used to dissociate biomass into cellulose, hemicelluloses and lignin bioproducts are often require extreme and expensive techniques. Although some ionic liquids were found to succeed as solvents using milder conditions, ionic liquids at large scale application still show limitations in terms of recoverability and cost [4]. Despite their high efficiency in dissolution of cellulose, new green approaches towards the replacement of volatile organic solvents were taken into consideration.

A new family of solvents which is known as "deep eutectic solvents (DES)" was presented for the first time by Abbott et al. as suitable alternative for ionic liquids [5]. However, this name does not cover all the class of solvents because many of them show glass transition points instead of eutectic melting points in which can be known as "low transition temperature mixtures (LTTMs)" [6]. These new solvents can be formed by mixing two solid starting materials which form a liquid through hydrogen bond interactions. They show interesting properties as solvents to be explored for different applications in separation processes. They share many advantages with ionic liquids but their preparation is simple and cheap. However, the building principles are not easy to generalize. Unlike normal chemical bonds, hydrogen bonds present different contact distances and binding energies which do not depend only on the donor and acceptor nature [7]. 